\title{
Limitations of In Vitro Assessments of the Drug Interaction Potential of Botanical Supplements
}

\author{
Authors \\ John S. Markowitz ${ }^{1,2,3}$, Hao-Jie Zhu ${ }^{1,2}$ \\ Affiliations \\ ${ }^{1}$ Department of Pharmacotherapy and Translational Research, University of Florida, Gainesville, Florida, USA \\ ${ }^{2}$ Center for Pharmacogenomics, University of Florida, Gainesville, Florida, USA \\ ${ }^{3}$ Center for Drug Interaction Research and Education, University of Florida, Gainesville, Florida, USA
}

Key words

- botanical

interaction

in vitro

in vivo

- metabolism

received April 12, 2012

revised June 12, 2012

accepted June 14, 2012

\section{Bibliography}

DOI http://dx.doi.org/

10.1055/s-0032-1315025

Published online July 18, 2012

Planta Med 2012; 78:

1421-1427 (c) Georg Thieme

Verlag KG Stuttgart - New York

ISSN 0032-0943

\section{Correspondence}

John S. Markowitz, PharmD

Department of Pharmaco-

therapy and Translational

Research

University of Florida College of

Pharmacy

1600 SW Archer Road,

RM PG-23

Gainesville, FL 32610-0486

USA

Phone: + 13522736262

Fax: + 13522736121

jmarkowitz@cop.ufl.edu

\section{Abstract}

$\nabla$

Although there are inherent and recognized limitations of in vitro screening methodologies to assess conventional drug-drug interactions (DDIs) per industry guidelines and those adopted by independent laboratories, further limitations are being appreciated which are unique to the evaluation of botanical products and potential DDIs in which they may participate. Among the larger issues faced are the uncertainty in assigning hepatic concentrations of multiple constituents and their potential metabolites, accounting for oral bioavailability, distribution, first-pass metabolism and active metabolites. Furthermore, the wide variability in the chemical composition of commercially available botanical supplement formulations continues to be a major concern, and manufacturing standards or enforcement thereof is essentially nonexistent in most countries. Differing formulations, unspecified product excipients, administration and absorption of the therapeutic ingredient(s) of a standardized dosage

\section{Introduction and Background}

$\nabla$

Approximately $50 \%$ of all licensed drugs that have been registered worldwide in the 25 year time period prior to 2007 were either natural products or synthetic derivatives [1]. It is now well recognized that the metabolism and disposition of conventional medications may be significantly influenced by the co-ingestion of various natural products and dietary supplements. These substances, widely used in both a complementary and alternative fashion, are inclusive of vitamins, minerals, herbal or other botanicals, amino acids or metabolic precursor substances, or combinations thereof. The present review will focus primarily on botanical dietary supplements and the limitations of widely utilized in vitro methods in the as- form, the very presence and/or concentration of one or more phytoconstituents within a supplement are typically unknown and nontarget entities. A further issue is the absence of authentic analytical standards, and the inability to accurately screen the entities as mixtures to even approximate typical scenarios, which may occur following the ingestion of dietary supplements, adds additional layers of complexity to experimental design and difficulty in interpreting experimental results. Multiple challenges exist in experimental methodologies employed in performing in vitro research with conventional pharmaceuticals and those unique to botanical extracts. These obstacles prevent the investigators from effectively utilizing high-throughput models to accomplish more than essentially "flag" suspected sources of drug interactions which must be further evaluated in vivo, at present, in order to confirm clinical significance. This review is intended to discuss the problems and challenges in evaluating botanical-drug interactions using in vitro methodologies.

sessment of botanical-drug interactions. Increasing interest in evaluating botanical-drug interactions is largely the result of the steady growth of dietary supplement use by consumers worldwide in the last 20 years - particularly in Western countries such as the US where up to $20 \%$ of the public reports regular usage [2].

Numerous products containing biologically active phytochemicals, so-called "secondary metabolites" are widely available for use worldwide. They are often termed "herbal" or "botanical" supplements and are derived from an enormous variety of plant sources. They can be purchased in numerous forms, the most common of which are encapsulated extracts. Extracts containing natural phytochemicals are also available as beverages and tinctures and can be ingredients in sports/nutri- 
tion drinks, powders, and "energy" bars. Functional food products containing specific phytochemical constituents are also being used by the public for the treatment of some common ailments [3]. Natural products do not have to be consumed as "medicines" to be involved in a drug interaction. A notable example is grapefruit juice, components of which (i.e., furanocoumarins) have been demonstrated in both in vitro investigations and in numerous clinical studies to increase the bioavailability of a variety of cytochrome P450 (CYP) 3A substrates [4]. Additionally, grapefruit juice as well as other natural health products may also inhibit other major phase I enzymes such as carboxylesterase (CES) [5-7]. Consumption of cruciferous vegetables (i.e., isothiocyanates) has been implicated in inducing the metabolism of a number of CYP1A2 substrates [8,9]. The potential influence of various natural products upon the metabolism and disposition of medications has been recognized for decades, well before the recent and drastic increase in use of these agents by the general public and subsequent reaction to the discovery that one of the most popular dietary supplements, St. John's wort (SJW), was firmly identified as a perpetrator in clinically significant botanical-drug interactions [10-12]. Thus, a generalization by the lay public that "natural means safe" soon gave over to the likewise over-generalization, that the SJW example must surely represent "only the tip of the iceberg" as an untold number of available botanical supplements were being combined with conventional drug therapies in an ever growing number of patients. Hence, a public health concern and awareness were born.

As a consequence, efforts to screen numerous botanical products for potential DDIs were soon underway with some sense of urgency given the widespread use among the general population often in those already prone to polypharmacy, e.g., elderly, HIV positive. These initial studies generally involved the more commonly used products and assessed their influence on major metabolic enzyme systems. As data accumulated, extensive lists of suspected botanical-drug interactions began to be generated based on emerging studies and specific phytoconstituents present in these products, the majority of which remain to be documented in a convincing manner $[10,11]$. There are numerous in vitro and in vivo methods available to screen botanical products for drug interaction potential, most being essentially a direct adaptation of methodologies applied to conventional medications in the assessment of DDI potential. Although many in vivo normal volunteer botanical-drug interaction studies have been and continue to be performed, the number of in vitro studies assessing the potential for drug interactions with natural products far exceeds the number of in vivo studies, and they remain the most cost effective and predominant type of research investigations performed to date. The use of animal models and human subjects in research on DDIs is generally limited due to a variety of factors including the associated costs of such investigations, interspecies differences that preclude the adequate predictive value of experiments, the availability of test subjects, feasibility of testing procedures, and ethical concerns about discomfort or pain caused to live subjects.

Although there are inherent and recognized limitations of in vitro screening methodologies to assess conventional drug interactions per industry guidelines and those adopted by independent laboratories, further limitations are being appreciated which are unique to the evaluation of botanical products and potential DDIs in which they may participate. Among the larger issues faced are the uncertainty in assigning hepatic concentrations of multiple constituents and their potential metabolites, accounting for oral bioavailability, and distribution including first-pass metabolism and active metabolites. Furthermore, the wide variability in the chemical composition of commercially available botanical supplement formulations continues to be a major concern and manufacturing standards or enforcement thereof are essentially nonexistent in most countries. Differing formulations, unspecified product excipients, typically compounds serving the primary purpose of aiding in the manufacture, administration and absorption of the therapeutic ingredient(s) of a standardized dosage form, and the very presence and/or concentration of one or more phytoconstituents within a supplement are typically unknown. Often supplements are standardized to a single phytoconstituent in the extract which is the putative active component, and in some cases that standardization "marker" may continue in spite of evidence emerging that it is not the component responsible for exerting therapeutic effects. Further issues are the absence of authentic analytical standards, and the inability to accurately screen the entities as mixtures to even approximate typical scenarios, which may occur following the ingestion of dietary supplements, and adds additional layers of complexity to experimental design and difficulty in interpreting experimental results.

Although many normal volunteer botanical-drug interaction studies have been performed and published, it is in vitro investigations assessing both potential for interaction as well as mechanisms by which various natural products may interact with metabolic enzymes and transporters which account for the majority of research performed and published to date. There are many commonly used methods to assess DDIs in vitro. These methods have strengths as well as limitations, and their use in screening conventional drugs has been more extensively reviewed elsewhere [13-15]. This limited review is intended to focus more on the particular problems and challenges in evaluating DDIs involving botanical supplements using in vitro methodology, and what can and cannot be inferred from the results of such investigations.

\section{Mechanisms of Drug Interactions}

$\nabla$

Drug interactions are generally classified as pharmacodynamic or pharmacokinetic. This review focuses mainly on pharmacokinetic interactions which occur when absorption, distribution, metabolism, or elimination are altered by another drug to produce the interaction. The primary route of drug elimination from the body is via biotransformation of the agent catalyzed by one or more enzymes [15]. Oxidative processes mediated by CYP450 enzymes are typically viewed as "functionalization" reactions said to represent phase I metabolism. In humans, approximately 70 active genes encode for various CYP450s of which CYP1A2, CYP2B6, CYP2C8, CYP2C9, CYP2C19, CYP2D6, CYP2E1, and CY$\mathrm{P} 3 \mathrm{~A} 4 / 5$ are considered the most relevant to the majority of conventional pharmaceuticals. Other phase I processes include metabolic reduction and hydrolysis. Conjugation reactions (phase II metabolism) typically follow phase I metabolism and involve the introduction of a hydrophilic endogenous species to the drug molecule, ultimately producing a more polar molecule which is more readily excreted in bile, feces, or urine. Glucuronidation is one such major pathway catalyzed by one of many uridine diphosphoglucuronosyltransferases [UGTs]. Other major metabolic pathways falling under the general phase II category include sulfation, methylation, acetylation, and glutathione conjugation. There are a significant number of drug-metabolizing enzyme (DME) families and superfamilies which may exhibit 
overlapping substrate specificities, and these properties are extensively reviewed elsewhere and well beyond the scope of the present review.

Phase I DMEs such as those comprising the CYP450 and serine hydrolase systems are found in essentially all tissues including the liver, intestine, lung, kidney, brain, adrenal gland, testes, heart, skin, and nasal mucosa. In the case of CYP3A4, the isoform is expressed in mucosal gut cells more abundantly than in the liver. In the liver [16], DMEs are primarily located in the endoplasmic reticulum of hepatic cells.

Enzymes involved in phase II reactions are mainly located in the cell cytosol, except UGTs, which are located in the endoplasmic reticulum. Furthermore, drug transporters such as P-glycoprotein (P-gp, $A B C B 1$ ), multidrug-resistance-associated proteins (MRPs, $A B C C$ ) and breast cancer resistant protein (BCRP, ABCG2) are increasingly appreciated with regard to their important roles in the disposition of many drugs, DDIs, and botanical-drug interactions $[17,18]$. There is clear in vitro evidence that numerous phytochemicals are potent inhibitors of $A B C$ transporters, although the exploitation of this property for the purposes of enhancing the therapeutic efficacy has yet to be realized in the clinical arena. Since many of the phytochemicals are charged [18], it stands to reason that both organic anion transporters (OATs), MRP2, organic cation transporters (OCTs), and P-gp are transporters of some of these compounds, and potentially may be inhibited by them as well.

The two major mechanisms of drug interactions involving enzymes and/or transporters are metabolic inhibition and induction. Metabolic inhibition can occur with almost all enzymes and transporters. Inhibitory effects on a specific drug metabolizing system can lead to toxicity as a result of increased plasma concentrations of the substrate compound and may occur as early as the first dosages administered depending on the agents involved. Toxicity may also occur if a previously minor metabolic pathway becomes favored, resulting in a toxic metabolite. Enzyme inhibition can also result in reduced clinical efficacy if the substrate is a prodrug [3,7]. Current opinion and regulatory guidance generally holds that studying inhibition of CYP1A2, CYP2C8, CYP2C9, CYP2C19, CYP2D6, and CYP3A4/5 enzymes is of most importance from a clinical perspective. Conversely, sufficient induction CYP 450 increases drug clearance which may lead to therapeutic failure $[11,19,20]$. Metabolic induction does not occur with all enzymes and transporters and is presently viewed as most significant for CYP3A, CYP1A2, as well as P-gp substrates. Metabolic induction of expression of DMEs by xenobiotics, including clinical therapeutics, is mediated by activation of the nuclear receptors constitutive androstane receptor (CAR) and/or pregnane X receptor (PXR). Primary human hepatocytes are considered to be a preferred in vitro model for the study of drug metabolism, especially metabolic induction relative to the models based on immortalized cells lines, such as HepG2 cells. However, primary hepatocyte models do exhibit some unique limitations. For example, the expression and activity of specific DMEs in primary hepatocytes could vary significantly between different donors due to genetic variation, pathological state, and varied techniques involved in tissue collection, storage, and transfer. Additionally, the expression of DMEs in primary hepatocytes may change over time in culture depending on in vitro culture conditions. All these factors may affect the predictability and reproducibility of primary human hepatocytes models. There are, however, recognized problems in detecting CAR-mediated enzyme induction as it is constantly activated in immortalized cells even before xenobiotic activation. This situation makes in vitro assessment of hCAR activation extremely challenging.

With transporters in general, inhibition and induction can lead to both an increase or decrease in plasma/active site concentrations depending upon substrate specificity, the direction of transport with respect to the active site of the drug and the specific type of transporter affected. Thus prediction of drug interactions through changes in transport interaction must take into consideration the direction of transport with respect to the individual drug and active site(s). Further, the interplay between metabolism and transport is only now beginning to be investigated.

\section{In Vitro Methods of Predicting Drug Interactions: Strengths vs. Weaknesses \\ $\nabla$}

The investigation into the DDI potential of a new chemical entity which may represent a promising candidate for clinical development has been a long-standing area of interest and investigation. Withdrawal of medications such as terfenadine, astemizole, cisapride, and mibefradil from the market demonstrate the risk of DDIs which had gone largely unrecognized prior to their approval by the US Food and Drug Administration (FDA). Drug interaction studies for new drug applications (NDAs) filed between 1987 and 1991 were largely in vivo studies with potential coadministered drugs, whereas for NDAs submitted between 1992 and 1997, the majority of studies involved metabolic mechanisms and in vitro methodology [21]. Despite current limitations in the extrapolation of in vitro drug metabolism data to the in vivo environment, in vitro studies remain the mainstay of initial evaluations, largely due to the high throughput nature of these investigations and the substantially reduced costs relative to in vivo studies [21,22]. Studies are typically carried out utilizing high-throughput in vitro study paradigms to assess compounds for DDI potential. The FDA has recently reported an increase in the inclusion of in vitro data in NDAs and acknowledges the importance of these data in evaluating these applications and eventual product labeling [21, 22 ]. Thus, the use of in vitro studies is clearly a valuable tool in drug development continuing to evolve in both methodology and interpretation. Although in vitro screening methods are both standard and accepted procedures in research and development efforts within the scientific community and pharmaceutical industry, a number of limitations are recognized in both the manner in which data are generated and how the extrapolation of such data to the in vivo situation is attempted on the compounds studied, metabolic pathway assessed, and system utilized [3]. Preclinical evaluation of drug metabolism and disposition as well as drug interaction assessments utilize a variety of in vitro enzyme and drug transporter sources and methodologies including hepatic and intestinal microsomes, liver homogenates, S9 fractions, cDNA expressed individual CYPs, or more purified enzyme preparations, primary hepatocytes, liver slices, and immortalized cell lines. Often cell lines that are deficient in, or overexpress a specific transporter such as P-gp may be utilized [22].

Although these methods are clearly more rapid and less expensive than animal and clinical studies, inherent limitations exist in both the methodology used and the utilization of generated in vitro data. Chief among these include the arbitrary assignment of drug concentration at the enzymatic and/or transporter site, the difficulty accounting for or even estimating presystemic metabolism, and the contribution of both known and unknown metabolites $[3,14,18,23]$. 


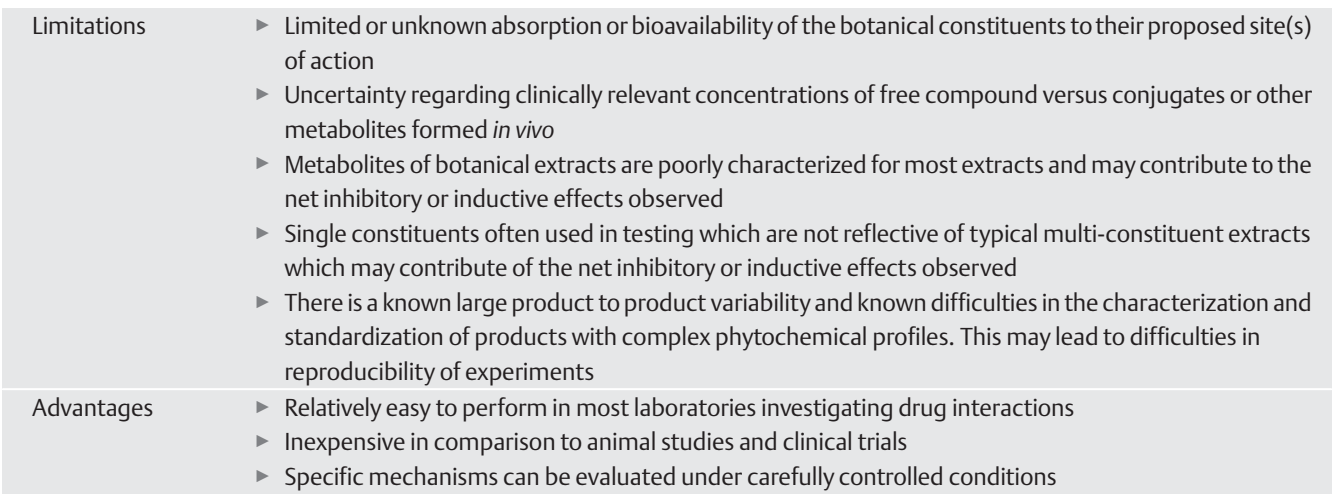

Table 1 General limitations and advantages of in vitro botanicaldrug interaction studies.

\section{Limitations of In Vitro Studies Specific to Botanical Supplement Assessment}

All of the aforementioned limitations must be monitored and carefully considered for appropriate interpretation of in vitro studies in extrapolating to interactions in humans. Nonetheless, in vitro studies still provide much value in identifying specific botanical products or constituents that may potentially pose an interaction risk and thus serve as a "signal" that in vivo studies may be warranted to confirm clinical relevance. In addition to the general limitations of in vitro studies which are noted above, due to the nature of these multi-constituent, nonstandardized, and generally not fully characterized products which make up botanical extracts and dietary supplements, unique challenges and caveats exist in the methods utilized in their evaluation as well as the interpretation of published reports. An overview of these limitations as well as advantages of in vitro study of botanical supplements is presented in 0 Table 1. Unlike uncooked fruits and vegetables wherein the degree of phytochemical exposure is dependent on the quantity ingested, exposure in botanical supplements is dependent on the dose, frequency, and formulation and is in theory, quantifiable [24]. In the case of the dietary supplement industry in which little manufacturing oversight is required, this could prove to be yet another source of variability leading to difficulties in interpretation of data. Botanical products contain a wealth of naturally occurring phytochemicals including alkaloids, xanthines, coumarins, terpenes, steroids, lipids, carotenoids, flavonoids, isoflavones, isothiocyanates, phenolic, cinnamic and amino acids, as well as di- and tripeptides [23,24]. This list is not to be construed as exhaustive as each class encompasses a wide variety of derivatives and stereoisomers, which expands further upon being subject to presystemic metabolism. In fact the differential absorption and bioavailability of stereoisomers has scarcely been investigated although there is precedent for drastic differences in the inhibitory activity of naturally derived stereoisomers quinine and quinidine relative to CYP2D6 and drug transporters $[25,26]$. It would be virtually impossible to perform meaningful clinical studies with most individual constituents. Thus, screening with in vitro methodologies has been a feasible method of predicting which botanical/dietary supplements may be involved in interactions with conventional drugs and warrant further "confirmatory" normal volunteer studies to assess true clinical relevance [3].

The selection of relevant concentrations of botanical supplements to assess in in vitro study systems is far more difficult than that of assessing conventional compounds as there is often little to no data published on what systemic concentrations are reached for phytoconstituents, and almost none that have been replicated. Indeed, the assignment of physiologically relevant concentrations for even the most thoroughly characterized conventional medication is a well-known limitation of in vitro studies; for almost all botanical products, there is little to no adequate pharmacokinetic data available on any but the most studied product. Furthermore, even these products which have undergone some formal study are limited to dose and formulation specific investigations, typically measuring only a few "marker" constituents that are characteristic of the product. These compounds may or may not represent the current prevailing thought on the purported active constituents. However, a given supplement may continue to be standardized based upon the content of an irrelevant compound while contents of "nonstandardized" constituents vary widely between proprietary products and perhaps within manufactured lots [27]. An accurate assessment of pharmacokinetic data is particularly problematic at present given the lack of comprehensive human disposition studies using well characterized botanical supplements. Of the small number of studies that are available for review, overall conclusions are limited for a number of reasons including use of different formulations, different or unknown dosages, and differences in what is measured analytically [3]. A problem frequently encountered in the existing in vitro literature is the use of inappropriately high concentrations of single isolated constituents obtained from commercial sources when only a small fraction of the compound may actually be bioavailable. Most natural products are generally subject to first-pass metabolism and to a much larger extent than conventional pharmaceutical agents which are in most cases specifically developed to be substantially bioavailable or otherwise formulated as prodrugs [3]. In addition, many are less bioavailable due to their hydrophilic nature or large molecular size. A number of recent studies assessing the biological activities of many phytochemical constituents of botanical products in vitro have been criticized due to the failure of investigators to acknowledge that these constituents do not exist when the native compounds are given in vivo but rather are present as conjugated metabolites that are formed primarily in the small intestine [3, 28 ]. Since unconjugated compounds do not ever reach the liver in many cases in any appreciable quantity, in vitro investigations to assess and predict the effects of these compounds on hepatic metabolism and transport are inaccurate [29].

The role of metabolites is almost entirely unknown for most supplements, not only with regard to any therapeutic contribution or participation in DDIs, but the very existence as of yet uncharacterized compounds. The potential for unknown or uninvestigated constituents or metabolites to influence a given enzyme 
or transporter of interest cannot be excluded from consideration. Further, the limited commercial availability of many phytochemicals and their principal metabolites precludes their initial screening using in vitro systems. Even if single constituents or metabolites were available, examining them as individual entities in vitro would be unlikely to be representative of the in vivo situation following ingestion of these supplements [3]. Plant extracts contain a mixture of compounds which are often structurally related and have similar biological properties. When consumed in a mixture they may have additive or antagonistic activities, which may not be apparent when an entity is tested in isolation.

The use of high concentrations of single constituents in in vitro study models when in fact the botanical extract is a complex mixture can result in the in vitro system being exposed to an exaggerated and unrealistic concentration. Additionally, if hepatic enzymatic or transporter activity is of interest, the issue of bioavailability needs to be closely considered, independent of first pass potential. If a compound accesses the portal circulation and is thus exposed to the liver, hepatic enzymes are theoretically vulnerable to whatever activity the compound might have. Extensive metabolism might result in low amounts of the compound reaching the central compartment. If there are other issues such that the compound never gets through the intestinal wall, the only enzymes or transporters that might be affected would be intestinal [e.g., CYP3A, P-gp]. Additionally, the variable content of specific constituents, variability between what are ostensibly the same products with regard to plant sources, quality control and lot to lot variability, recommended dosage, dose frequency, and duration of dosing regimens make extrapolations of in vitro findings to the in vivo situation exceedingly difficult [3, 13]. With multiple and structurally similar components found in most botanical extracts, these extracts may be particularly susceptible to the generation of "false" positive/negative results through some laboratory assay. For example, spurious data could be generated if a fluorescent probe is used in a DDI study which involves the detection of a compound(s) with autofluorescent or fluorescence quenching properties. This may be especially problematic when the analytic assay is not capable of differentiating individual compounds (e.g., a method based on microplate reader determinations). This issue can be circumvented through careful evaluation of the methodology and the use of appropriate probes or a more selective analytical method (e.g., HPLC, LC-MS).

\section{In Vitro Findings Versus In Vivo Studies in Humans}

The discrepancies between in vitro studies and the in vivo DDI study are well illustrated by the example of milk thistle [Silybum marianum (L.) Gaertn. (Fam. Asteraceae)]. Milk thistle botanical supplements purportedly exert hepatoprotective effects via components found within the extract of milk thistle seeds, called silymarin, which are a variable mixture composed of the flavonolignans silybin (also termed silibinin) A \& B, isosilybin, silydianin, silychristin, and taxifolin. Most milk thistle supplements are standardized to contain at least $70 \%$ silymarin. Utilizing human in vitro study methodologies including both microsomal and hepatocyte assays following exposure to what were believed to be physiologically relevant concentrations of silymarin and its components has been found to produce inhibitory effects on the metabolism of CYP3A and CYP2C9 substrates by a number of independent investigators [30-32], although Zuber and associates
(2002) reported that inhibition of CYP3A did not occur at relevant concentrations [33]. Additionally, there is in vitro evidence of inhibition of the phase II [conjugative] enzymes UGT 1A6/9/1 [31] and P-gp [34,35]. Furthermore, following the oral feeding of silybin to mice, significant induction of two phase II enzymes was reported, glutathione S- transferase and quinone reductase, respectively [36].

However, when formal drug interaction study protocols were undertaken in healthy volunteers, at least three published studies appear to exonerate milk thistle extracts from participating in any significant metabolic interaction involving CYP3A or P-gp. First, Piscitelli and coworkers [37] detected no interaction between milk thistle supplements containing silymarin and the protease inhibitor and CYP3A/P-gp substrate, indinavir. Similarly, employing a different study design, DiCenzo and coworkers found no influence of milk thistle supplementation on indinavir concentrations [38]. Gurley and associates determined that milk thistle supplementation in normal volunteers reduced midazolam (CYP3A probe substrate) clearance only minimally [39]. Lastly, our group also determined there to be no significant effect upon midazolam pharmacokinetics (administered as part of a probe drug cocktail) following exposure to the proprietary milk thistle supplement Legalon ${ }^{\circledR}$ (Markowitz, unpublished data).

The reason for these conflicting results in in vitro and in vivo findings with silymarin or one or more of its components are not readily apparent. All in vivo studies utilized different milk thistle supplements, and it is well recognized that silymarin supplements differ substantially from one product to another [40]. Additionally, the in vivo microenvironment where DDIs occur differs significantly from the conditions (i.e., incubation buffer) employed in the in vitro studies, and consequently, biological responses of enzymes and transporters to silymarin as well as other xenobiotics could be different between in vitro and in vivo studies. Furthermore, like most in vitro studies, the aforementioned milk thistle experiments did not take into account the high plasma protein binding rate of silybin, which was approximately $70 \%$ according to a previous report [41]. A possible scenario is that the inhibition of isolated CYP3A in vitro could in fact be fully compensated for in vivo by alternative metabolic pathways which are unaffected by silymarin. Finally, after oral administration, silymarin is rapidly metabolized to conjugated metabolites by sulfatases and $\beta$-glucuronidases, to the extent that plasma concentrations of conjugated metabolites far exceed the concentrations of free parent compounds [42]. Thus, the discrepancies of the in vitro and clinical findings may be in part due to the presence of a high concentration of silymarin metabolites following biotransformation in vivo.

\section{Other Considerations}

$\nabla$

\section{Solvents utilized in in vitro studies may adversely}

influence the integrity of the assay

Researchers may arbitrarily dissolve the product in a solvent or buffer which may contain dimethyl sulfoxide (DMSO), ethanol, or other organic solvent. The solubility of the constituents is likely to further contribute to experimental variability. Some phytoconstituents are inherently difficult to solubilize. For example, lycopene, a carotenoid with potent antioxidant effects and abundant in red tomatoes, watermelon, pumpkin, plum, persimmon, and many other fruits in which it imparts a reddish color is of great research interest. However, it is essentially insoluble in 
water, sparingly in ethanol, methanol, cyclohexane, and DMSO, soluble in ethyl ether and acetone, and freely soluble in chloroform and benzene. Further, like many phytoconstituents, it is unstable and may readily oxidize at room temperature. Thus, placing the compound into cell culture for metabolism or transport studies can prove extremely difficult to accomplish while still maintaining cell viability by avoidance of toxic solvents.

\section{Derived cell lines must be carefully chosen based upon a specific assay and substrates}

The cell type/line utilized in in vitro studies may overexpress certain enzymatic proteins that adversely affect drug metabolism in numerous ways. For example, the Caco-2 cell line derived from heterogeneous human epithelial colorectal cells. Caco-2 cells are most commonly used not as individual cells, but as a confluent monolayer on a cell culture filter (e.g., Transwell). When cultured in this manner, the cells differentiate to form a polarized epithelial cell monolayer that provides a physical and biochemical barrier to the passage of ions and small molecules. The Caco-2 monolayer is extensively utilized as an in vitro model of the human small intestinal mucosa in order to predict the absorption of orally administered drugs. The correlation between the in vitro apparent permeability across Caco- 2 monolayers and the in vivo fraction absorbed is well established. Caco-2 cells express tight junctions, microvilli, and a number of enzymes and transporters that are characteristic of such enterocytes including P450 enzymes, peptidases, esterases, P-gp, and some uptake transporters for amino acids, bile acids, carboxylic acids, and others. However, such cell lines may also prove to be liabilities in studying some pharmacological or investigative agents due to their overexpression of specific enzymes, transporters, and other proteins. For example, Caco-2 cells were found to be largely inappropriate for studying the metabolism or transport of human carboxylesterase 1 (hCES1) dependent drug substrates (e.g., methylphenidate) or prodrugs (e.g., oseltamivir) due to the overexpression of hCES1 within this cell line [43]. A further consideration is the observed variability and heterogeneous nature of Caco-2 cells which may differ significantly between laboratories and even form different subclones during culture. For these reasons, quantitative comparisons of data generated by different laboratories have to be performed with caution and with recognition of these potential confounders.

\section{Pharmaceutical excipients}

When performing in vitro or in vivo assessments of drug interaction potential, seldom appreciated is the potential influence of some excipients, generally regarded as inert, which are added into various oral dosage formulations. Common examples include lactose, microcrystalline cellulose, starch, stearate lubricants, sorbital, and others. Although excipients are generally believed to be devoid of any ability to disturb the PK and/or PD of "actives" they are routinely combined with, in certain cases, the concentration and disposition of certain medications may be influenced. Excipients have many functional groups that may interact with specific pharmaceutical entities $[44,45]$.

\section{Conclusions}

$\nabla$

In vitro methods have been widely used in attempts to predict potential drug interactions with botanical products, but to date, few significant drug interactions have actually been discovered or ac- curately predicted with botanical products. Instead, much of the research has led to clinical studies with negative outcomes. While the exoneration of a given botanical product is an important finding and may produce some measure of comfort to clinicians, the expense and effort to perform these studies is not trivial.

In vitro studies utilized to assess drug interaction potential with natural products have all the limitations of in vitro studies with conventional drugs including assignment of hepatic concentrations, accounting for first-pass metabolism and active metabolites. Additionally, the lack of commercial standards, and the inability to accurately screen the entities as mixtures remain a strategic interest [46]. These complications have contributed to the discrepancies between outcomes predicted by in vitro results and observed in vivo effects.

Cell-based models are probably the method of choice for in vitro investigation of transporter-mediated DDIs since intracellular substrate accumulation cannot be determined in non-cell-based models. However, non-cell-based models, such as cell membrane preparations, could prove to be very useful for rapid assessment of the affinity of a specific transporter to its substrates. For DMEsmediated DDI, non-cell-based systems such as hepatic and intestinal microsomes, s9 fractions, and purified individual enzymes are more rapid and also more applicable to the development of a high-throughput screening assay. However, one limitation of non-cell-based models is the inability of such models to take into account the potential interplay of drug transporters and DMSs. For example, a liver microsome incubation study might suggest a tested drug is a potent inhibitor of a specific CYP 450 enzyme. However, in vivo, such a DDI may never occur if the inhibitor is a substrate of an efflux transporter (e.g., P-gp, BCRP) that prevents the inhibitor from reaching the requisite concentration to cause enzyme inhibition.

Other high-throughput approaches including the use of in silico models have been increasingly utilized to evaluate the potential dispositional characteristics of even theoretical compounds. Although in vitro approaches are clearly more rapid and less expensive than clinical studies, and may generate an early signal of drug interaction potential or provide insight into the mechanistic aspects of DDIs, inherent limitations are recognized in all available in vitro models. These include perhaps most prominently the difficulties in the assignment of hepatic concentrations reflective of likely in vivo scenarios due to a variety of sources of variability, the lack of clinical pharmacokinetic data of numerous pharmacologically active constituents of a given herbal product, and the difficulty accounting for first-pass metabolism, the potential production and contribution of metabolically active metabolites and others. Accordingly, it may be time to reevaluate the value of in vitro screening of individual phytoconstituents and extract mixtures and critically assess the clinical implications from in vitro studies that are conducted, with consideration to the many limitations.

\section{Acknowledgements \\ $\nabla$}

R21 AT002817-01: NIH NCCAM

\section{Conflict of Interest}

$\nabla$

The authors report no conflicts of interest. 


\section{References}

1 Newman DJ, Cragg GM. Natural products as sources of new drugs over the last 25 years. J Nat Prod 2007; 70: 461-477

2 Bailey RL, Gahche JJ, Lentino CV, Dwyer JT, Engel JS, Thomas PR, Betz JM, Sempos CT, Picciano MF. Dietary supplement use in the United States, 2003-2006. J Nutr 2011; 141: 261-266

3 Markowitz JS, von Moltke LL, Donovan JL. Predicting interactions between conventional medications and botanical products on the basis of in vitro investigations. Mol Nutr Food Res 2008; 52: 747-754

4 Greenblatt DJ, von Moltke LL, Harmatz JS, Chen G, Weemhoff JL, Jen C, Kelley $\mathrm{CJ}$, LeDuc BW, Zinny MA. Time course of recovery of cytochrome p 4503 A function after single doses of grapefruit juice. Clin Pharmacol Ther 2003; 74: 121-129

5 Li P, Callery PS, Gan LS, Balani SK. Esterase inhibition by grapefruit juice flavonoids leading to a new drug interaction. Drug Metab Dispos 2007; 35: 1203-1208

6 Li P, Callery PS, Gan LS, Balani SK. Esterase inhibition attribute of grapefruit juice leading to a new drug interaction. Drug Metab Dispos 2007; 35: 1023-1031

7 Liu R, Tam TW, Mao J, Saleem A, Krantis A, Arnason JT, Foster BC. The effect of natural health products and traditional medicines on the activity of human hepatic microsomal-mediated metabolism of oseltamivir. J Pharm Pharm Sci 2010; 13: 43-55

8 Rodriguez-Fragoso L, Martinez-Arismendi JL, Orozco-Bustos D, Reyes-Esparza J, Torres E, Burchiel SW. Potential risks resulting from fruit/vegetable-drug interactions: effects on drug-metabolizing enzymes and drug transporters. J Food Sci 2011; 76: R112-R124

9 Ioannides $C$. Effect of diet and nutrition on the expression of cytochromes P450. Xenobiotica 1999; 29: 109-154

10 Chavez ML, Jordan MA, Chavez PI. Evidence-based drug-herbal interactions. Life Sci 2006; 78: 2146-2157

11 Piscitelli SC, Burstein AH, Chaitt D, Alfaro RM, Falloon J. Indinavir concentrations and St John's wort. Lancet 2000; 355: 547-548

12 Mannel M. Drug interactions with St John's wort:mechanisms and clinical implications. Drug Saf 2004; 27: 773-797

13 von Moltke LL, Greenblatt DJ, Schmider J, Wright CE, Harmatz JS, Shader $R I$. In vitro approaches to predicting drug interactions in vivo. Biochem Pharmacol 1998; 55: 113-222

14 Pelkonen O, Turpeinen M, Uusitalo J, Rautio A, Raunio H. Prediction of drug metabolism and interactions on the basis of in vitro investigations. J Basic Clin Pharmacol Toxicol 2005; 96: 167-175

15 Wienkers $L C$, Heath TG. Predicting in vivo drug interactions from in vitro drug discovery data. Nat Rev Drug Discov 2005; 4: 825-833

16 Dürr D, Stieger B, Kullak-Ublick GA, Rentsch KM, Steinert HC, Meier PJ, Fattinger K. St John's Wort induces intestinal P-glycoprotein/MDR1 and intestinal and hepatic CYP3A4. Clin Pharmacol Ther 2000; 68 : 598-604

17 Lin JH, Yamazaki M. Role of P-glycoprotein in pharmacokinetics: clinical implications. Clin Pharmacokinet 2003; 42: 59-98

18 Li Y, Revalde JL, Reid G, Paxton JW. Interactions of dietary phytochemicals with $\mathrm{ABC}$ transporters: possible implications for drug disposition and multidrug resistance in cancer. Drug Metab Rev 2010; 42: 590611

19 Ruschitzka F, Meier PJ, Turina M, Luscher TF, Noll G. Acute heart transplant rejection due to Saint John's wort. Lancet 2000; 355: 548-549

20 Alfaro CL. Emerging role of drug interaction studies in drug development: the good, the bad, and the unknown. Psychopharmacol Bull 2001; 35: 80-93

21 Masimirembwa CM, Thompson R, Andersson TB. In vitro high throughput screening of compounds for favorable metabolic properties in drug discovery. Comb Chem High Throughput Screen 2001; 4: 245-263

22 Kosugi Y, Hirabayashi H, Igari T, Fujioka Y, Hara Y, Okuda T, Moriwaki T. Evaluation of cytochrome P450-mediated drug-drug interactions based on the strategies recommended by regulatory authorities. Xenobiotica 2012: 42: 127-138

23 Gurley BJ, Hagan DW, editors. Herbal and dietary supplement interactions with drugs. Boca Raton: CRC Press; 2003

24 van Breemen RB, Fong HHS, Farnsworth NR. Ensuring the safety of botanical dietary supplements. Am J Clin Nutr 2008: 509S-513S

$25 \mathrm{Hedman}$ A, Meijer DK. The stereoisomers quinine and quinidine exhibit a marked stereoselectivity in the inhibition of hepatobiliary transport of cardiac glycosides. J Hepatol 1998; 28: 240-249
26 Ai C, Li Y, Wang Y, Chen Y, Yang L. Insight into the effects of chiral isomers quinidine and quinine on CYP2D6 inhibition. Bioorg Med Chem Lett 2009; 19: 803-806

27 Kroon PA, Clifford MN, Crozier A, Day AJ, Donovan JL, Manach C, Williamson $G$. How should we assess the effects of exposure to dietary polyphenols in vitro? Am J Clin Nutr 2004; 80: 15-21

28 Gurley BJ. Emerging technologies for improving phytochemical bioavailability: benefits and risks. Clin Pharmacol Ther 2011; 89: 915-919

29 Donovan JL, DeVane CL, Boulton D, Dodd S, Markowitz JS. Dietary levels of quinine in tonic water do not inhibit CYP2D6 in vivo. Food Chem Toxicol 2003; 41: 1199-1201

30 Sridar C, Goosen TC, Kent UM, Williams JA, Hollenberg PF. Silybin inactivates cytochromes P450 3A4 and 2C9 and inhibits major hepatic glucuronosyltransferases. Drug Metab Dispos 2004; 32: 587-594

31 Venkataramanan R, Ramachandran V, Komoroski BJ, Zhang S, Schiff PL, Strom SC. Milk thistle, a herbal supplement, decreases the activity of CYP3A4 and uridine diphosphoglucuronosyl transferase in human hepatocyte cultures. Drug Metab Dispos 2000; 28: 1270-1273

32 Beckmann-Knopp S, Rietbrock S, Weyhenmeyer R, Böcker RH, Beckurts $K T$, Lang $W$, Hunz $M$, Fuhr $U$. Inhibitory effects of silibinin on cytochrome P-450 enzymes in human liver microsomes. Pharmacol Toxicol 2000; 86: 250-256

33 Zuber R, Modriansky M, Dvorák Z, Rohovský P, Ulrichová J, Simánek V, Anzenbacher $P$. Effect of silybin and its congeners on human liver microsomal cytochrome P450 activities. Phytother Res 2002; 16: 632638

34 Zhang S, Morris ME. Effect of the flavonoids biochanin A and silymarin on the P-glycoprotein-mediated transport of digoxin and vinblastine in human intestinal Caco-2 cells. Pharm Res 2003; 20: 1184-1191

35 Zhang S, Morris ME. Effects of the flavonoids biochanin A, morin, phloretin, and silymarin on P-glycoprotein-mediated transport. J Pharmacol Exp Ther 2003; 304: 1258-1267

36 Zhao J, Agarwal R. Tissue distribution of silibinin, the major active constituent of silymarin, in mice and its association with enhancement of phase II enzymes: implications in cancer chemoprevention. Carcinogenesis 1999; 20: 2101-2108

37 Piscitelli SC, Formentini E, Burstein AH, Alfaro R, Jagannatha S, Falloon J. Effect of milk thistle on the pharmacokinetics of indinavir in healthy volunteers. Pharmacotherapy 2002; 22: 551-556

38 DiCenzo R, Shelton M, Jordan K, Koval C, Forrest A, Reichman R, Morse G. Coadministration of milk thistle and indinavir in healthy subjects. Pharmacotherapy 2003; 23: 866-870

39 Gurley BJ, Gardner SF, Hubbard MA, Williams DK, Gentry WB, Carrier J, Khan IA, Edwards DJ, Shah A. In vivo assessment of botanical supplementation on human cytochrome P450 phenotypes: Citrus aurantium, Echinacea purpurea, milk thistle, and saw palmetto. Clin Pharmacol Ther 2004; 76: 428-440

40 Venkataramanan $R$, Komoroski B, Strom S. In vitro and in vivo assessment of herb drug interactions. Life Sci 2006; 78: 2105-2115

41 Wu JW, Lin LC, Hung SC, Chi CW, Tsai TH. Analysis of silibinin in rat plasma and bile for hepatobiliary excretion and oral bioavailability application. J Pharm Biomed Anal 2007; 45: 635-641

42 Wen Z, Dumas TE, Schrieber SJ, Hawke RL, Fried MW, Smith PC. Pharmacokinetics and metabolic profile of free, conjugated, and total silymarin flavonolignans in human plasma after oral administration of milk thistle extract. Drug Metab Dispos 2008; 36: 65-72

43 Imai T, Imoto M, Sakamoto H, Hashimoto M. Identification of esterases expressed in Caco-2 cells and effects of their hydrolyzing activity in predicting human intestinal absorption. Drug Metab Dispos 2005; 33: $1185-1190$

44 Pifferi G, Restani P. The safety of pharmaceutical excipients. Farmaco 2003; 58: 541-550

45 Chen ML, Straughn AB, Sadrieh N, Meyer M, Faustino PJ, Ciavarella $A B$, Meibohm B, Yates $C R$, Hussain AS. A modern view of excipient effects on bioequivalence: case study of sorbitol. Pharm Res 2007; 24: 73-80

46 Xin GZ, Qi LW, Shi ZQ Li P, Hao HP, Wang GJ, Shang J. Strategies for integral metabolism profile of multiple compounds in herbal medicines: pharmacokinetics, metabolites characterization and metabolic interactions. Curr Drug Metab 2011; 12: 809-817 